\title{
Paroles de témoins : Vers une pluralisation du récit historique
}

\author{
Words of Witnesses: Towards a pluralization of \\ the historical narrative
}

\section{Las palabras de los testigos: Hacia una pluralización de la narrativa histórica}

\author{
Danièle Tosato-Rigo \\ Université de Lausanne, Switzerland
}

\begin{abstract}
RÉSUMÉ
Le retour de l'individu et du singulier dans l'écriture de l'histoire constitue l'un des changements majeurs qu'a connus l'histoire socioculturelle depuis les années 1980. Réhabilitée, après une longue période de règne de narratifs macrohistoriques et d'histoire sérielle, l'approche biographique en est sortie élargie, transformée en une donnée incontournable de la compréhension des contextes sociaux. En revenant sur ce changement de paradigme, cet article se focalise sur deux courants ayant ouvert de nouvelles perspectives qui pourraient trouver d'ultérieurs développements en histoire de l'éducation : la recherche sur les " egodocuments » et la microhistoire (" Microstoria »). Il analyse ensuite à titre d'exemple un "fait éducatif » local et non spectaculaire - l'institutionnalisation d'un séminaire de formation de maîtres et de maîtresses, au milieu du XVIIIe siècle - à travers les traces qu'il a laissées dans les archives.
\end{abstract}

Mots clés: histoire socioculturelle, histoire de l'éducation, époque moderne, egodocuments, microhistoire, formation des maîtres-ses.

\section{ABSTRACT}

The return of the individual and the singular in the writing of history is one of the major changes in socio-cultural history from the 1980s onward. The biographical approach has been rehabilitated after a long period in which the macro-historical narratives and the serial history dominated. The biographical approach emerged, enlarged and transformed, as an inseparable part of the understanding of social contexts. Going back to this change of paradigm, this article focuses on two currents that opened new perspectives that could be further developed in history of education: the research of "egodocuments," and the microhistory. As an example, 
I analyze a local, non-spectacular "educational case" - the institutionalization of a formative course for teacher educators in the mid eighteenth century - by examining the traces that have been left in archives.

Key words: socio-cultural history, historiography and history of education, modern times, egodocuments, microhistory, teacher education.

\section{RESUMEN}

La vuelta del individuo y de lo singular en la escritura de la historia es uno de los mayores cambios en la historia sociocultural desde los años ochenta en adelante. La aproximación biográfica ha sido rehabilitada después de un largo periodo de tiempo en el que las narrativas macrohistóricas y la historia serial fueron dominantes. La aproximación biográfica surge ampliada y transformada en una parte inseparable de la comprensión de los contextos sociales. Volviendo a este cambio de paradigma, este artículo se centra en dos corrientes que abren nuevas perspectivas las que podrían ser más desarrolladas en la historia de la educación: la investigación de "egodocumentos" y la "microhistoria." Como ejemplo, yo analizo un caso educativo local que no es espectacular, la institucionalización de un curso formativo para profesores de mediados del siglo XVIII mediante los huellas que han quedado en los archivos.

Descriptores: historia sociocultural, historiografía e historia de la educación, tiempos modernos, egodocumentos, microhistoria, formación de profesores.

$S^{\prime}$ IL FALLAIT TRADUIRE EN UNE IMAGE UNE ÉVOLUTION HISTORIOGRAPHIQUE à mes yeux majeure de ces dernières décennies dans le domaine de l'histoire socioculturelle de l'époque moderne (XVIe-début XIXe s.), je l'esquisserais volontiers sous la forme d'un portrait collectif. On y verrait réunis quatre personnages : un pasteur anglais, un compagnon vitrier parisien, un meunier et un métayer du nord de l'Italie. Tous quatre, respectivement Ralph Josselin, Jacques Louis Ménétra, Domenico Scandella - dit Menocchio - et Gian Battista Chiesa, furent tirés de l'anonymat dans les années 1980 à la faveur d'une tendance historiographique mettant en avant les acteurs ordinaires de l'histoire. ${ }^{1}$

"Ordinaires », ces personnages devenus historiques l'étaient dans un double sens. D'un côté par leur qualité d'homme du commun, éloigné des grandes figures de l'histoire, et de l'autre par leur vie telle qu' elle intéressait les historiens : une trajectoire biographique insérée dans les choses banales de l'existence, avec son lot d'événements répétitifs et peu spectaculaires.

Tandis qu'Alan Macfarlane (1970) a proposé avec Ralph Josselin une approche anthropologisante, Daniel Roche (1982) inscrivait Ménétra dans une histoire sociale de la culture, et Carlo Ginzburg (1980) posait avec Giovanni Levi (1989) les premiers jalons de ce que l'on appellera la microhistoire. Au-delà de la diversité de leurs approches, tous quatre s'inscrivaient dans le même moment de contestation des récits macrohistoriques et de l'histoire quantitative (transformée par les débuts de l'informatique). Une perspective qui n'a rien perdu de son actualité, quand bien même la "culturalisation " de l'histoire et une distance prise entretemps avec l'histoire sociale a atténué l'intérêt particulier que la génération fondatrice avait porté aux humbles et aux marginaux, au profit d'un plus large spectre d'individus, provenant de toutes les couches sociales. 
Issus de cette période féconde de réinvention de l'archive et de l' " humanisation des sciences sociales " (Dosse, 1995), deux courants ont, parmi d'autres, contribué à ouvrir de nouvelles perspectives de recherches qui pourraient trouver d'ultérieurs développements en histoire de l'éducation, conçue comme partie intégrante de l'histoire socioculturelle. C'est du moins ce qu'entend suggérer cet article, qui s'articulera en deux parties : la première se penchera sur les avancées de la recherche sur les « egodocuments » et sur les remises en cause apportées par la microhistoire. Dans la seconde partie, je donnerai un exemple, fondé sur un travail en archives, destiné à montrer l'intérêt de repenser le local et le particulier par rapport au "global ».

\section{Le retour de I'individu}

Dans les années 1970, deux modèles interprétatifs dominent dans l'historiographie française - à laquelle la revue des Annales et les historiens qui s'y associent donnent une posture importante sur la scène internationale (Walter, 1997, p. 63). D'une part celui de type macroéconomique, indifférent aux acteurs (notamment par réaction contre l'histoire historisante), qui use de l'analyse quantitative et sérielle ; et, d'autre part, pour tout ce qui échappe à l'analyse en termes de structures économiques et sociales, celui qui s'appuie sur le concept flou de mentalités. Mais l'affaiblissement des grandes certitudes des Annales et l'essoufflement de ces modèles heuristiques dominants pendant une vingtaine d'années (1960-1980) inaugurent un moment de flottement qui favorise le retournement. Ce qu'Antoine Prost a appelé « l'effondrement du paradigme labroussien ", en référence à l'histoire macroéconomique d'Ernest Labrousse et de ses successeurs, s'est également caractérisé par une remise en cause de l'idée même qu'un historien puisse proposer quelque chose comme un point de vue de surplomb sur la réalité historique (Prost, 2012). En pleine autocritique, la revue des Annales offre en 1989 ses colonnes à Giovanni Levi pour une réflexion sur la biographie. L'historien la prône comme le lieu idéal pour vérifier le caractère interstitiel - et néanmoins important - de la liberté dont disposent les agents historiques, comme pour observer la façon dont fonctionnent concrètement des systèmes normatifs qui ne sont jamais exempts de contradictions.

Si le retour de l'individu perceptible dans l'historiographie dès les années 1980 marque la réhabilitation du biographique, il faut comprendre ce dernier dans une définition considérablement élargie. Des personnages "secondaires " ou même microscopiques ont rejoint les politiciens, militaires ou artistes. Sans compter la nouvelle prise en compte des femmes. En outre, les "nouvelles fortunes de la biographie ", pour reprendre R. Pasta (2009, p. X-XI) en font une donnée complémentaire de la compréhension des contextes sociaux, autant qu'un laboratoire du vécu dans lequel se décantent les stratégies et motivations du choix individuel.

\section{Recherche sur les " egodocuments " et nouvelles pistes}

Le renouveau d'intérêt pour l'individuel, dans cette perspective élargie, a stimulé dans divers pays européens dès les années 1980 la recherche sur les " egodocuments " : un terme, comme celui de 1st Person Writing, conventionnel dans la recherche internationale (traduit par "Selbstzeugnisse » en allemand, « Ecrits du for privé » ou « Ecrits 
personnels » en français) regroupant les autobiographies, mémoires, journaux, livres de raison, correspondances et autres documents rédigés volontairement et d'un point de vue personnel. La recherche, dont l'étendue ne rend plus guère possible le survol, s'articule notamment autour de la relation entre histoire et mémoire, des rapports " moi-individu-société », de la transculturalité, des attitudes religieuses, du rapport à la santé et au corps et des pratiques éducatives. ${ }^{2}$

Nonobstant le caractère empirique de ces travaux - qui a fait dire à James Amelang que les chercheurs concernés n'avaient pas encore tiré toutes les conclusions de l'élargissement de l'autobiographique (Amelang, 2007) - on peut relever deux transformations importantes intervenues de façon générale dans le traitement du témoignage, en partie influencées par le linguistic turn, sans pour autant avoir conduit à une approche strictement discursive. En premier lieu, on ne recherche plus la représentativité, ni d'une vision du monde ni d'une catégorie sociale (Tosato-Rigo, 2009, p. 1214). Et pas davantage la fameuse "exception normale " selon la formule d'Edoardo Grendi (1972). En collectant des cas singuliers, parfois organisés sous forme de " panels » (Hanafi, 2012), la tendance actuelle vise à retrouver la diversité de l'éventail social, et à faire d'eux des « lieux d'observation des possibles qu'offrait l'espace social à un ou plusieurs individus" (Ruggiu, 2005, p. 12).

Une attention accrue est dévolue dans cette investigation à la parole du témoin. Kaspar von Greyerz (2010, p. 44) a fait remarquer à ce propos, en justifiant la longueur de certaines citations reproduites dans son ouvrages sur les âges de la vie, que contrairement à la façon de procéder dans l'historiographie plus ancienne, qui se contentait souvent de fragments de citations arrachées à leur contexte, il s'agit bien d'incorporer en les citant une partie du contexte que les écrits personnels véhiculent. Car c'est aussi leur force d'expression au sens plus large qui importe, et donc leur textualité. L'heure n'est plus à opposer texte et contexte. Par ailleurs, libéré en quelque sorte de l'obligation de représentativité, l'écrit du for privé n'est plus - seconde transformation - considéré comme un simple réservoir d'informations, quand bien même on peut encore, en l'historicisant et en le contextualisant, y puiser quelques informations inédites sur le monde historique (Ulbrich, 2007). C'est un creuset d'expérience, à même de rendre compte de l'agentivité (" agency ") des scripteurs, et c'est aussi une production de la culture écrite, qui mérite d'être étudiée en tant que telle.

Le potentiel des écrits du for privé pour l'étude de l'enfance a été mis en avant dès la fin des années 1970 (Hardach-Pinke, 1981; Hardach-Pinke \& Hardach, 1978, Pollock, 1988; Schlumbohn, 1983; Seibel, 1986). Toutefois nombre de travaux qui les ont exploités se sont limités à en reproduire des extraits à titre illustratif. Une prospection à la fois plus intensive et plus approfondie de cette documentation offre de nouvelles possibilités. Elle semble en particulier susceptible d'apporter des solutions à la difficulté régulièrement relevée (Becchi \& Julia, 1998) d'appréhender une éducation domestique qui concerne jusqu'au milieu du XIXe siècle un nombre bien plus élevé de familles que l'éducation - mieux étudiée - en établissements publics ou privés, et qui s'avère la principale voie d'instruction pour les filles. Le journal tenu jour par jour par la gouvernante de Cécile de Constant, étudié par Sylvie Moret Petrini (2010), a par exemple permis d'observer les connaissances transmises à cette 
jeune fille de la haute bourgeoisie lausannoise dès l'âge de six ans et jusqu'à ses seize ans, ainsi que le contrôle familial et social dont elle a été l'objet. Le caractère genré, non seulement de l'éducation dispensée, mais des responsabilités éducatives peut être investigué : Pierre Caspar (1996) a ainsi, sur la base de correspondances familiales neuchâteloises, émis l'hypothèse d'une féminisation de l'autorité éducative au XVIIIe siècle, tandis Martine Sonnet (1990) a sollicité des sources françaises analogues pour analyser les modèles éducatifs paternels et les tensions résultant de la pression sociale.

L'historiographie de l'éducation pourrait profiter davantage de la recherche sur les egodocuments, non seulement parce que cette dernière a depuis longtemps noué l'histoire de l'éducation à celle de l'enfance, comme à celle de la famille dont Stefan Ehrenpreis (2010) a relevé à quel point il était important de mieux connaître l'implication éducative, mais encore en raison de son intérêt pour l'étude des liens entre théories et pratiques.

Rudolf Dekker (2002) en a fait la démonstration dans le cas hollandais, en analysant des egodocuments pour mieux comprendre la réception des idées pédagogiques. S'agissant d'allaitement ou de systèmes de punitions, entre autres, l'historien a pu ainsi mettre en évidence l'existence d'un fossé insoupçonné entre les idées des pédagogues et les pratiques familiales. La fine analyse à laquelle l'historien a soumis, avec Arianne Braggerman, le journal d'un adolescent néerlandais, Otto van Eyck, dessine les contours de l'éducation que reçoit ce fils de bonne famille comme le reflet d'une pratique influencée par les Lumières (Braggerman \& Dekker, 2009). De son côté, la thèse en cours de Sylvie Moret Petrini (2010) reconstitue à plus large échelle - celle de la Suisse romande - la «boîte à outils pédagogiques " propre aux protagonistes de l'éducation (géniteurs, parenté, éducateurs professionnels, mais aussi enfants) grâce à une approche fondée sur l'étude contextualisée des écrits personnels. Une entreprise qu'il conviendrait d'étendre géographiquement et chronologiquement. De même qu'il serait utile de recenser et d'analyser, autant pour leur regard sur eux-mêmes que sur leurs pratiques éducatives, les écrits personnels d'éducateurs/ trices et d'instituteurs/trices, à l'instar du journal de l'instituteur saint-gallois Gregor Grob (1754-1824) qui, à l'aide de signes, faisait la somme des points obtenus, en comportement et en apprentissage, par ses élèves à la fin de chaque mois (Leutert \& Piller, 1999, p. 211). L'analyse par Jaquet-Francillon (2005) de récits de vie rédigés par des instituteurs au XIXe siècle, qui interroge la notion d'identité professionnelle, fournit un cadre analytique utile à ce propos, en distinguant leur situation, leur statut professionnel et leur fonction sociale, tels que les scripteurs les perçoivent, les subissent ou les revendiquent.

Dans la perspective d'une histoire de l'expérience (Erfahrungsgeschichte), l'étude des processus d'apprentissage trouve des matériaux particulièrement intéressants dans les egodocuments. Songeons à l'autobiographie du paysan suisse Ulrich Bräker (Bürgi, 1998-2010), ou à celle de Valentin Jamerey-Duval (Goulemot, 1981), berger lorrain devenu bibliothécaire d'un prince, autodidactes - le premier apprend à lire dans le cercle piétiste, le second avec d'autres bergers - que le patronage d'un plus puissant qu'eux amène à dépasser les frontières assignées à leur savoir par les conventions sociales. Le façonnement des comportements, ou encore les modalités de la 
socialisation constituent autant d'autres pistes à suivre (Caspard, 1996, 2006; Moret Petrini, 2010; Piller, 2001; Tosato-Rigo, 2014). D'autant que, contrairement à ce qui a été généralement retenu, journaux, mémoires et autobiographies n'émanent pas uniquement de scripteurs d'âge moyen, voire avancé, mais de plus jeunes, y compris d'enfants. Quant à la provenance sociale des scripteurs, elle inclut aussi le « milieu de la société " et plus exceptionnellement des représentants des couches populaires (Amelang, 1998; Duran Lopez, 2009). Le fait que des acteurs de position, sexe et âges différents tiennent la plume pluralise le discours éducatif et le miroir qu'ils nous tendent de l'éducation, domestique ou non.

\section{L'expérience de la " microstoria $\|^{3}$}

La reconsidération de la conception du rôle de l'individu dans l'historiographie a suscité, conjointement à la biographisation de l'histoire, ce que l'on a appelé une " microhistoire " dont le foyer fut la revue italienne des Quaderni Storici, autour d'historiens tels que Carlo Ginzburg, Giovanni Levi, Carlo Poni, Edoardo Grendi et Maurizio Gribaudi. Tandis que le premier, avec l'histoire de Menocchio, lui donnait une orientation culturelle, le second, avec son ouvrage Le Pouvoir au Village (1989) privilégiait l'orientation sociale, qui trouvera davantage d'échos dans le paysage historiographique. Les récents travaux de Simona Cerutti (2008) montrent qu'une telle division n'est pas inhérente à l'approche microhistorique : cette dernière pourrait même relier histoire sociale et histoire culturelle.

Comme l'a souligné Jacques Revel (1989, p. XI-XII) les "microhistoriens » suggéraient une réponse à une question très concrète : celle de savoir comment introduire l'histoire sociale dans une historiographie italienne marquée par l'idéalisme (de Benedetto Croce) et par des contraintes universitaires limitant les chantiers collectifs, sans entrer dans la dépendance de modèles historiographiques importés, principalement de France ou du monde anglo-saxon.

Dans les traits caractéristiques de la microhistoire italienne de cette période fondatrice, le principal est sans nul doute - davantage que la réduction de l'espace d'investigation - le procédé de changement d'échelles. Il implique un rapport inventif à la réalité historique par le biais de la variation systématique de la focale et de l'angle d'observation. Il nécessite l'adaptation continuelle de l'outil d'analyse à l'objet qu'il étudie, objet qui doit être constamment redéfini. Outre ce mouvement dynamique, et comme l'a relevé Jacques Revel (1994, p. 554), la microhistoire peut aussi s'éloigner momentanément de son objet premier - ou même en changer en cours de route - par des excursus apparemment fort éloignés de l'objet qui, reliés entre eux, apporteront un éclairage nouveau.

On touche là à l'un des points centraux de la microhistoire, en lien direct avec sa critique d'une méthode de l'histoire sociale "traditionnelle »: l'application d'hypothèses fonctionnalistes préétablies qui ne débouchent pas toujours sur une explication satisfaisante. La microhistoire, au contraire, "fait bouger les images reçues, parce qu'en réglant différemment la distance et l'ouverture de leur optique, les observateurs font apparaître une autre trame, des découpages différents, et du même coup l'inadéquation partielle des outils conceptuels dont ils disposaient jusque-là » (Revel, 
1989, p. XV). Autrement dit, on sait d'où l'on part, mais (pendant longtemps), pas où l'on arrive et encore moins par où l'on passe. Une autre critique adressée par les microhistoriens à l'histoire sociale "traditionnelle " étant celle de dresser une ou des barrières entre le texte et l'historien, ou plus généralement la source et l'historien, et d'empêcher celui-ci de percevoir des aspects qui ne rentrent pas dans le cadre des hypothèses préétablies.

En mettant l'accent sur les angles d'observation, la microhistoire assume pleinement la subjectivité du chercheur. Elle assimile à certains égards son travail à celui du cinéaste. Un rapprochement qui n'est pas fortuit : Carlo Ginzburg s'est expliqué dans la revue Critical Inquiry (1993) sur l'influence qu'ont exercé sur lui les travaux du sociologue du cinéma Sigfried Kracauer membre du cercle « Poetik und Hermeneutik » au milieu des années 60, qui réunissait entre autres Reinhart Koselleck et Hans Robert Jaus, et dont le travail sur l'histoire n'a commencé à être reçu que dans les années 1990 (Despoix \& Schöttler, 2006). Ce que l'on a appelé depuis les « jeux d'échelle " historiographiques constitue l'un des thèmes récurrents du livre (posthume) de Kracauer. Histoire: Les Avant-dernières Choses qui analysait les tensions existant entre approche macro- et micro-historique, avec une préférence marquée pour la seconde et pour la valorisation du détail, mais sans jamais renoncer aux circulations entre les deux niveaux, comme le feront aussi les microhistoriens.

Le caractère expérimental de l'approche de ces derniers ressort clairement de l'ouvrage phare de Giovanni Levi, Le Pouvoir au Village. Il se décèle dès son sous-titre. Celui-ci annonce l'histoire d'un exorciste dans le Piémont du XVTIe siècle. Or l'histoire de l'exorciste en question, Giovanni Battista Chiesa, n'occupe qu'une petite partie de la recherche. Il disparaît même pour ne revenir qu'à la fin de l'enquête à laquelle il sert de point de départ, voire de prétexte. La reconstitution de l'histoire de G. B. Chiesa n'intéresse pas Levi pour elle-même. S'il l'a choisie c'est parce que « c'est précisément cette dimension quotidienne de l'histoire vécue par un groupe de personnes, impliquées dans des événements locaux mais liées à des faits politiques et économiques échappant à leur contrôle direct, qui posent des problèmes riches de suggestion sur les motivations et les stratégies de l'action politique ". L'historien récuse les modèles généraux que tente de construire l'histoire économique et sociale globalisante et les définitions qui cherchent "à rassembler les comportements en une seule loi explicative. " (Lévi, 1989, p. 10). Par le retour au "singulier» (en l'occurence les trois familles de métayers du village de Santena que l'historien passe au peigne fin dans les archives locales), Giovanni Levi met en cause le concept de la naissance de l'Etat moderne, ${ }^{4}$ la longue durée, la conception traditionnelle de la famille : des cadres interprétatifs inopérants dans le cas de Santena - mais qui donnent néanmoins sens à ce dernier - parce qu'ils ne prennent pas en considération la discontinuité, "l'instabilité des préférences individuelles, des ordres institutionnels, des hiérarchies et des valeurs sociales : en résumé, le processus politique qui engendre le changement, mais aussi les directions imprévisibles prises par celui-ci, fruit de la rencontre entre des protagonistes actifs."

La microstoria n'est ni une théorie de l'histoire ni même une méthode, mais, après avoir été une expérience (et les expériences ne sont pas destinées à durer) une 
perspective, qui combine des éléments bien connus de l'analyse historique. Et qui pourraient être utilisés dans d'autres approches, fussent-elles même globalisantes, pour autant qu'elles s'intéressent aux changements d'échelle, comme l'a souligné Jacques Revel (1996). C'est bien ce qui fait d'elle une sorte d'instrumentaire mobilisable pour d'autres questions que celles auxquelles ses fondateurs ont tenté de répondre. Et notamment, comme j'aimerais l'illustrer par l'exemple qui suit, pour des questions intéressant l'histoire de l'éducation. Plus que les stratégies des acteurs, c'est la mise en lumière de leur "culture pragmatique " (Cerutti, 2008) qui me semble particulièrement intéressante : les comportements, les actions des individus enregistrés dans les sources archivistiques qui légitiment ces dernières et constituent " des interprétations de ce que la structure sociale aurait dû être» (p. 151).

\section{Local vs. global : un exemple lausannois}

Le fait autour duquel s'organise cette brève enquête est révélé par le rapport imprimé d'une société philanthropique de Lausanne. La petite bourgade suisse d'un peu plus de 8'000 habitants - siège de la première académie protestante francophone d'Europe - était sous l'Ancien Régime le chef-lieu d'un bailliage faisant partie, comme tout le Pays de Vaud, de la République de Berne. Voici ce que l'on peut lire dans l'un des rapports officiels de ses Ecoles de charité :

"Le Sieur Guignard, premier Régent [= maître d'école], ayant par un effet ordinaire de son zèle, présenté à la Société en janvier 1757 un mémoire tendant à former un séminaire de jeunes régents et de jeunes maîtresses d'école, en étendant et en augmentant ce qui a déjà été fait à cet égard depuis longtemps, [...] la Société, après la circulation du projet, l'examen qui en fut fait et les modifications qui y furent apportées, l'approuva extrêmement ». (Etat, 1757)

Le fait en lui-même, cet aval donné par une société philanthropique à une formation d'enseignant-e-s, est peu spectaculaire, on en conviendra. Il est rendu à peine plus intriguant par la date à laquelle il a lieu : soit une trentaine d'années avant que des formations de maîtres d'école ne commencent à être mises en place en Suisse, et encore, uniquement pour le sexe masculin. Sans compter que l'initiative en question se révèlera durable : jusqu'à la création de la première école normale du canton, en 1833, les Ecoles de charité de Lausanne détiendront le monopole de la formation institutionnelle des enseignants vaudois.

Quelles légitimations portent les traces laissées par ce " fait éducatif »? Quelles attentes révèlent-elles ? Enfin, qu'apporte l'exemple lausannois, en changeant d'échelles d'observation, à l'histoire de l'éducation ? Autant de questions mettant en jeu le pouvoir, le savoir et la communication, auxquelles je chercherai à apporter quelques éléments de réponse.

\section{Charité, éducation et pouvoir}

Le projet de former des maîtres et des maîtresses s'inscrit dans le contexte de la création des "Ecoles de charité " lausannoises (en réalité une seule école, comprenant 
plusieurs classes). Celles-ci sont fondées le 14 mai 1726 par une assemblée de notables, constituée sous le nom de "Société Charitable ", avec l'appui gouvernemental. Ses membres, appelés « contribuants», finançaient sous forme de parrainage, dons et legs, la scolarisation d'enfants. A leur tête, et élus par cette assemblée, douze membres issus du corps professoral de l'Académie de Lausanne et de la magistrature locale, proches - par ascendance ou mariages - des milieux du Refuge huguenot : huit ecclésiastiques et quatre laïcs. Une différence au demeurant moins importante qu'on pourrait le penser, au vu de l'engagement confessionnel des laïcs. C'est davantage leur orientation religieuse qui distinguait les membres fondateurs, en l'occurrence leurs liens plus ou moins étroits avec le courant du christianisme libéral incarné notamment par Jean-Frédéric Ostervald.

En se référant à l'exemple des Charity schools anglaises promues par la Society for Promoting Christian Knowledge (SPCK), avec laquelle ils étaient en contact, les initiateurs des écoles lausannoises se proposaient de soustraire les enfants indigents entre huit et seize ans à l'«irréligion " en leur donnant une éducation appropriée et quelques secours (en pain, vêtements etc.). La démarche, à Lausanne comme à Londres, participait d'une vaste campagne des mœurs entreprise pour lutter contre la « crise morale " que les initiateurs diagnostiquaient dans leur époque (Shoemaker, 1992). A cette éducation religieuse s'ajoutait dans la ville vaudoise une formation professionnelle : non seulement une occupation par le travail manuel (notamment dans une « fabrique de coton »), comme dans les Workhouses, mais un apprentissage dans une profession susceptible d'assurer la subsistance des élèves à leur sortie de l'école.

La SPCK avait investi le champ de l'éducation populaire pour assurer la reconnaissance du primat alors contesté de l'église anglicane (Unwin, 1984). Le projet de la Société charitable lausannoise s'inscrit lui aussi dans une dynamique de pouvoir, quoique différente. Un conflit majeur venait en effet d'opposer les professeurs de l'Académie à leur souverain. Le gouvernement bernois leur avait imposé la signature d'un formulaire de foi (Formula Consensus) pour asseoir le respect de l'orthodoxie face aux courants piétistes, arminiens et autres dissidences. La mesure avait suscité une vive résistance, allant jusqu'à la démission d'un certain nombre de professeurs, dont le philosophe de renom Jean-Pierre de Crousaz. Ce sont précisément les opposants à la Formula Consensus - les théologiens Théodore Crinsoz, Gabriel Bergier et Georges Polier - que l'on retrouve parmi les initiateurs des Ecoles de charité.

L'appui gouvernemental apporté à la création de ces écoles, à la fois symbolique et matériel (livraisons de vins, grains, et ultérieurement de subventions), avait quelque chose d'une mesure compensatoire. Une fois la souveraineté réaffirmée, le pouvoir négocié reprenait sa place. Il reconnaissait aux principaux protagonistes (et perdants) du conflit une marge de manœuvre qui permettait à ces notables de reprendre par le biais d'un projet éducatif une forme de leadership. Et ce à deux niveaux. D'une part, l'instruction religieuse pourrait s'y développer, avec le consentement tacite du souverain, dans le sens du christianisme libéral - le maître d'école nommé en 1727 reçoit une Bible « avec les réflexions de M. Ostervald » - et les Ecoles mettront ultérieurement cette autonomie à profit pour éditer du matériel scolaire (psaumes, prières, catéchisme et liturgie pour l'enseignement). D'autre part, la Société charitable 
bénéficiait d'un droit de réunion au moment même où défense était faite au clergé vaudois de réunir ses délégués en synodes et aux principales villes du Pays de Vaud de tenir des assemblées communes.

Une décennie seulement après sa création, soit fin 1737, la Société a déjà produit non moins de vingt-sept règlements pour assurer la gestion de l'établissement et l'organisation de l'instruction, sur des questions aussi diverses que la scolarité obligatoire ou les rapports avec les maîtres d'apprentissage. Renforcé par l'adoption d'un système administratif tout droit issu du modèle urbain, qui génère son lot de dicastères et de responsables (le président pour les écoles, celui pour la fabrique, le directeur des livres, ceux pour l'habillement, la distribution de pain, les apprentissages, le directeur catéchiste ou l'inspecteur en bâtiments par exemple), le caractère para-étatique de la Société saute aux yeux. De fait, cette dernière prend en charge une mission qui n'est assumée ni par l'Etat, ni par la ville de Lausanne, qui n'aide que les enfants de bourgeois, et ne dispose que de quatre écoles de quartier.

La Société charitable combine les caractéristiques d'une association religieuse avec celles d'une société éclairée, au sens où les a définies Thomas Maïssen (2002). Son assemblée générale, réunie à l'hôtel de ville, met en délibération la gestion des écoles - l'introduction du travail, par exemple, comme l'institution du séminaire pour enseignant-e-s. Elle publie des rapports, annuels et quinquennaux, de ses activités et de ses comptes. ${ }^{5}$ Non nominatifs, ils ne permettent pas d'identifier les courants qui s'y affrontent. Mais ils rendent compte de débats qui la divisent et interpellent le public : sur la question du " trop » de savoir qui pourrait parvenir aux enfants des classes inférieures, par exemple, ou sur le type de métiers convenant à des enfants indigents, tout comme sur la répartition entre connaissances (religieuses) et savoir pratique. Curieusement, la formation des enseignant-e-s ne donne lieu à aucun débat.

\section{Former un chrétien utile à la société}

Les traces laissées par le projet initial de formation des maîtres sont peu nombreuses. On a le sentiment de se heurter à la règle de l'évidence : pourquoi mettre par écrit ce qui va de soi, ce qui fait consensus? Sans compter que dans ce microcosme lausannois où les fondateurs étaient également liés entre eux par des liens de parenté, la communication devait être essentiellement orale.

Il faut revenir, pour en entendre parler, à l'organe de communication de la Société. Au moment où paraît son troisième rapport imprimé, le public visé par la campagne des mœurs a largement répondu à l'appel, puisque les Ecoles de charité accueillent cent trente-huit enfants, également répartis entre les deux sexes. Un succès favorisé par la gratuité de l'enseignement et de ses supports (livres, plumes, encre, ouvrages de piété), qui s'ajoutent à l'aide en vêtements et en pain. La Société s'en félicite tout en annonçant son projet de former des maîtres d'école :

"Nous nous proposons même et nous espérons d'établir un séminaire de régents bien formés et instruits dans la religion et la bonne méthode pour la faire entendre au peuple, dont tout le pays a un très grand besoin " (Etat, 1728, p. 11). 
Premier constat : le projet, qui se limite au sexe masculin, dépasse clairement le cadre de la cité qui l'a vu naître. Une campagne d'évangélisation ne pouvait être envisagée qu'à l'échelle d'un territoire : c'est tout son peuple qu'il s'agissait de sauver. Second constat : la Société cultive une ambition pédagogique. La «bonne méthode » évoquée dans l'extrait ci-dessus est illustrée dans le rapport. Elle prévoyait l'introduction d'un procédé destiné à mesurer individuellement les progrès des élèves dans les matières enseignées, ${ }^{6}$ les mœurs et le " génie " : avec la "grande feuille ", à remplir trimestriellement par les Directeurs en charge des visites d'écoles (Etat, 1726-1732, p. 6). De telles grilles d'évaluation (puisque c'est bien de cela qu'il s'agit) complétées ne nous sont parvenues que pour les années 1780 (cf. Tosato-Rigo, 2012). Le contrôle trimestriel et individuel de l'apprentissage des psaumes, comme de la fréquentation (" assiduité ") des écoles est par contre attesté par quelques feuilles complétées pour les années 1751 à 1759 .

La Société charitable entend former un chrétien, mais un chrétien utile à la société, inspiré par une éthique du travail typique du protestantisme (et du puritanisme) et non dénuée d'utilitarisme :

"Il est certain qu'une bonne et chrétienne éducation est très nécessaire, pour former les enfants des pauvres à la piété et à la vertu, et pour leur inspirer l'amour du travail. Il n'est pas moins certain que la piété, la vertu, et l'amour du travail, sont d'une nécessité absolue aux personnes pauvres, non seulement pour être heureuses dans ce siècle et dans le siècle à venir ; mais aussi pour assurer le repos et la tranquillité des autres hommes, de quelques conditions qu'ils soient ; puisqu'il n'y a personne qui ne puisse retirer quelques services des pauvres, s'ils sont honnêtes gens, ou souffrir quelque mal de leur part, s'ils manquent de probité. "(Relation, 1726, p. 1-2)7

Le prosélytisme religieux rendait nécessaire la formation de maîtres : les Ecoles se donnaient les moyens de les façonner, de leur inculquer les règles et la doctrine de l'institution et d'en faire des agents de leur diffusion. Elles en forment d'abord pour elles-mêmes. Une fois le premier maître et la première maîtresse engagés, elles recrutent systématiquement le personnel enseignant parmi leurs élèves. L'ouverture d'une troisième classe est ainsi rendue possible parce qu'un des élèves " s'est trouvé en état d'enseigner séparément " (Etat, 1727-1728, p. 11).

M. G. Jones a évoqué à propos des écoles de charité britanniques la « naissance d'un nouveau maître d'école élémentaire » (Jones, 1938, p. 96-97), dont l'inspiration piétiste - avec le modèle de Francke à Halle - n'est pas à négliger : c'est le " maître à plein temps ", alors inexistant dans les petites écoles publiques. Il a à sa disposition un curriculum, une méthode d'enseignement et des règles précises que la SPCK a commandées au vicaire de Spofforth James Talbot, sous la forme du Christian Schoolmaster (1707). Ainsi que des dispositions plus concises et organisationnelles qui figurent dans les Methods Used for Erecting Charity Schools : la Société charitable lausannoise s'en inspire largement. Elles règlent notamment le nombre d'heures de leçons, les compétences à développer chez les élèves et comportements à réprimer, ainsi que 
l'encadrement religieux par les maître-sse-s aux catéchismes publics.

On peut s'étonner de l'absence de normes écrites relatives à la formation des élèves-régents. Les deux premiers candidats font leur apparition en 1728 : le rapport des Ecoles se borne à signaler qu'ils se forment auprès du maître principal. Cette pratique - qui pendant des décennies ne concernera guère plus d'un à deux élèves par an $\neg-$ est fixée dix ans plus tard seulement, dans un règlement général de la Société charitable sur les apprentissages. Il stipule que les futurs maîtres accompliraient cet apprentissage avec le régent de la grande école, " en profitant de toutes ses instructions et en le soulageant de ses fonctions, et qu'on leur fournirait en pain par semaine jusqu'à la concurrence de 3 écus blancs " (Etat, 1737-1742, p. 24). C'est trois décennies plus tard qu'un premier règlement distinct pour ces élèves précisera qu'ils bénéficiaient d'une heure de cours particulière, chaque matin, pour approfondir les différentes matières enseignées, voire, si nécessaire, d'une année supplémentaire de formation, pendant laquelle la Société charitable se chargerait, toujours si nécessaire, de leur entretien. Elle continuerait en outre à les pourvoir en pain, afin que le souci alimentaire ne vienne pas les distraire de l'étude (Règlement, 1767).

Entretemps, la direction des Ecoles de charité a commencé à inscrire la formation des maîtres dans un discours d'utilité publique :

«On n’a rien négligé pour guérir leurs [= des enfants indigents] mauvais penchants, pour les rendre assidus, les animer au travail. On a donné toute la faveur possible aux travaux de la terre, aux arts et aux occupations du ménage par un arrangement des heures, conforme aux saisons et autres circonstances. Lorsqu'on a vu des talents marqués pour l'instruction on les a cultivés et l'on a réservé quoiqu'en petit nombre les sujets propres à des régences, vu l'utilité d'un tel séminaire, pour remplir successivement les divers postes dans les villes et dans les villages de ce pays. " (Etat, 1747, p. 14)

En insistant sur l'utilité de ce qu' elle appelle déjà le " séminaire » de régents, la Société charitable visait moins à promouvoir ce dernier qu'à répondre aux critiques qui s'élevaient contre l'octroi d'apprentissages réputés « non pénibles " - selon la conception biblique du «dur labeur» - aux classes inférieures. Des critiques qui reviennent périodiquement. Elles sont renforcées par l'essor des idées physiocratiques et l'intérêt des cercles gouvernementaux bernois à améliorer le rendement agricole. Pourquoi ne pas fournir davantage de bras à l'agriculture ? Une argumentation à laquelle les magistrats et ecclésiastiques de la ville commerçante qu'était Lausanne, pourvue de vastes terres viticoles, se montraient peu sensibles. Le rapport imprimé de 1755 riposte avec la première statistique d'élèves :

"Le travail étant chose importante et les craintes que l'espèce d'éducation donnée ne rendit les élèves moins propres aux travaux publics, le public sera heureux d'apprendre que depuis 1727334 élèves sortants se sont voués à 60 professions différentes toutes utiles et la plupart pénibles, aidés en cela par quelque secours en argent lorsque cela a été nécessaire pour entrer en apprentissage. » 
(Etat, 1755, p. 15)

Avec 26 maîtres formés depuis 1727, qui ont - spécialité lausannoise ? - appris en même temps un métier manuel, le plus souvent cordonnier ou tisserand, la profession enseignante se situait dans le trio de tête du classement : après les vignerons (41) et les domestiques (36), mais avant les laboureurs (24), maçons (19), charpentiers (17), et tailleurs (12).

Pour ce qui est des filles, le rapport imprimé déclare qu' " un assez grand nombre d'entre elles se sont vouées au service domestique ou à l'économie de leur propre ménage» (p. 16). Le document manuscrit ayant servi de base à ce rapport disait toutefois autre chose. On y lit en effet que « la plupart [des filles] sont devenues gouvernantes, filles de chambre, maîtresses d'école, servantes et mères de familles, en état de bien élever leurs enfants." ("Liste des garçons ") Les filles étaient donc un certain nombre depuis 1727 à avoir effectué un apprentissage de maîtresses d'école. Mais loin de s'en vanter, la Société charitable censurait l'information, attestant par là des limites du consensus régnant sur la formation féminine et, surtout, celle de filles pauvres.

\section{Religion, pédagogie et didactique}

Dès le milieu des années 1740, les directeurs des Ecoles de charité réfléchissent à la façon d'encadrer les maîtres dans l'enseignement d'une religion qui ne peut selon leur christianisme rationnel se révéler opérante que comprise et intériorisée par les élèves. Ils envisagent la simplification de la liturgie, du nombre de psaumes à faire apprendre aux enfants (pour cesser de surcharger leur mémoire) et celle du catéchisme. Une démarche qui prévoit de donner également « quelques avis à nos maîtres et maîtresses, pour les aider à tourner leurs instructions de la manière la plus propre à éclairer les enfants » (Etat, 1744, p. 7; Etat, 1745, p. 11).

Deux documents isolés montrent le soin particulier apporté à l'engagement de nouveaux maîtres, mais également de maîtresses aux Ecoles de charité, et le profil attendu.

En 1745, deux candidates à un poste, filles de réfugiés huguenots et anciennes élèves des Ecoles, Jeanne Bourillon (24 ans) et Judith Favre (20 ans) doivent lire devant une commission de directeurs un chapitre du Nouveau Testament et sont interrogées (séparément) sur la 1ère partie du symbole des Apôtres avant de donner, quelques jours plus tard, une leçon en classe consistant à faire lire les enfants, à les faire prier, réciter quelques versets de psaume et quelques sections de catéchisme. Enfin, le lendemain, «le président leur a donné par écrit à chacune quelques questions de religion, pour y faire la réponse par écrit, sous les yeux de la 1ère maîtresse (des Ecoles), afin de connaître par là leur écriture ou orthographe et leur faculté à expliquer dans le besoin les vérités et les devoirs de la religion.» Le résultat des épreuves est exposé sur trois pages par le professeur Georges Polier, président ecclésiastique des Ecoles de charité. Il passe en revue les qualités et défauts des candidates dans cinq rubriques : lecture, religion, enseignement, écriture, conduite et mœurs, ouvrages (couture, dentelle, tricot). Avec profusion de détails, ${ }^{8}$ qui confirment l'attention portée par les examinateurs à la volonté et à la capacité des maîtresses de faire comprendre aux 
enfants ce qu'ils lisaient ou révisaient.

On retrouve le même souci dans les modalités de l'examen de cinq candidats pour deux postes de régents, qui porte "sur la lecture, la récitation, l'écriture, l'orthographe, l'arithmétique, le chant, une composition sur des questions de religion faite par chacun d'eux sous les yeux du comité, [... et sur la manière d'enseigner toutes ces choses, et en particulier la religion, à des enfants de différentes portées, que l'on amenait exprès des écoles. " (Etat, 1751). Là aussi, Polier rédige un long "Précis de mon jugement sur les épreuves des prétendants à la régence des écoles de charité ». En prévision de l'examen il avait dressé une liste de ce qu'il fallait exiger des candidats et candidates, insistant sur la nécessité de vérifier leur maîtrise des connaissances à transmettre "par jugement ", soit en faisant appel au raisonnement des enfants (Polier, 1750). Une méthode que deux candidats ne sont pas à même de mettre en pratique pendant leur leçon. ${ }^{10}$

On peut prendre la mesure de l'effort didactique entrepris au sein des Ecoles de charité en se tournant vers un autre genre de sources : les supports de l'enseignement religieux. Là encore, le professeur et apologétiste Polier, qui vient en 1747 de publier ses Pensées chrétiennes contre les Pensées philosophiques de Diderot (Marx, 1971), est l'âme et la plume de l'entreprise. Sa liturgie pour les Ecoles de charité (Polier, $1747)^{10}$ qui comprend des psaumes et des extraits de la Bible contient une explication introductive à chaque passage, reformulant ce qui précède et ce qui suit : un contexte utile au catéchiste, et dont on ne trouve pas l'équivalent, par exemple dans la Liturgie pour Neuchâtel établie en 1713 par Ostervald. (Dahn, 2013, p. 111). Quant à son Abrégé du Catéchisme d'Ostervald pour les Ecoles de Charité (Polier, 1750), qui sera réédité d'innombrables fois jusqu'au XIXe siècle, il constitue, comme l'a bien montré Nathalie Dahn, un véritable manuel avec ses 85 notes en caractères plus petits parsemant le texte, destinées aux éducateurs (parents compris), où sont expliquées - à l'intention d'enfants à partir de huit ans - les notions difficiles (telles " holocauste ", " intercession ", " manne » ou " amendement ") et proposés des exemples particulièrement éloquents de l'Histoire sainte. Dans cette didactique de la religion, l'attention des enseignants est attirée sur les aspects les plus divers de la transmission de connaissances, allant de l'importance de diviser la matière (" ou quelque réponse trop chargée ") à celle de ne pas faire répondre trop souvent les élèves par oui ou par non, " de peur qu'ils ne soient pas assez attentifs à ce qu'on leur demande " (Dahn, 2013, p. 105-113).

Malgré le fort lien qu'il est possible d'établir entre religion et pédagogie, les initiatives éducatives ne répondent pas au principe de convergence. Tout ne vise pas à l'intériorisation de la foi. Pourquoi, en 1752, un règlement introduit-il l'enseignement de l'écriture pour les deux sexes (ce qui différait des pratiques des écoles publiques, où l'écriture était réservée aux garçons avancés) ? Et pourquoi les directeurs des Ecoles soutiennent-ils au même moment l'apprentissage de la dentelle chez les filles plutôt que le filage "qu'on considère comme convenant mieux aux filles du peuple " avec l'argument que le filage " fournit moins de ressources aux filles pour entrer en service ou pour gagner leur vie avec le temps que la couture ou la dentelle " (Etat, 1752). Dans l'idée que le chrétien devait être utile à la société, c'était l'utilité à soi-même 
qui prévalait parfois. A moins qu'il ne faille y voir une demande de " contribuants " parrainant un enfant pauvre, qui souhaitaient peut-être, comme ses parents, avoir plus de chances de lui trouver un travail.

Si en 1757 - nous y revenons enfin - la Société charitable approuve l'institutionnalisation d'un séminaire d'enseignants pour élèves des deux sexes, c'est encore, vraisemblablement, à Polier qu' elle le doit. Selon les procès-verbaux des délibérations de la Direction des Ecoles le premier régent de l'école, Guignard, avait mis en circulation un projet d'établissement pour former " de jeunes régents ». Chargé d'y apporter des corrections, le professeur de théologie le modifia de façon substantielle en proposant «de faire un établissement pareil pour les filles, en y changeant ce qui conviendra ». On peut supposer que c'est la primauté de la tâche évangélisatrice qui, aux yeux de Polier, plaidait en faveur de la formation des maîtresses.

Le séminaire tel que présenté dans le rapport imprimé de 1757 était destiné à donner aux candidat-e-s à la régence des instructions " pour les former à l'enseignement clair et méthodique des vérités de la Religion ", avec une connaissance plus étendue de l'Histoire sainte et, "s'ils y sont propres ", de la musique d'église, de même que " pour les perfectionner dans la lecture, l'écriture et l'orthographe », et " les fortifier dans l'arithmétique " (Etat, 1757). La Société charitable qui, comme à ses débuts, en 1727, se prévalait d'une méthode pour enseigner la religion, revendiquait désormais clairement son monopole en ambitionnant d' "être en état de pourvoir successivement de régents et maîtresses les villes et communautés qui en demandent " (p. 17).

\section{Quelques questions plutôt que toutes les réponses}

Peut-on, en changeant d'échelle, mesurer quelques effets de la formation des maîtresse-s dispensée au sein des Ecoles de charité de Lausanne ? Et quelque intérêt à ce cas local, dans une perspective plus générale? Sans doute.

Un premier élément à relever est l'impact territorial, avec la demande que suscita un vivier qui dotait maîtres et maîtresses d'une " méthode », délivrait un certificat de capacité et qui s'était acquis une réputation, renforcée par le soutien gouvernemental et celui de la ville de Lausanne. Un séminaire qui exerçait de surcrô̂t les régents dans des fonctions publiques qu'ils étaient souvent appelés à remplir : conduire le chant des psaumes à l'église, y faire les prières, lire les commandements avant le service divin. Sans compter le métier manuel qu’ils y avaient appris, qui déchargeait les collectivités du souci d'augmenter les pensions trop chétives. Ou encore, qui exerçait les maîtresses à l'enseignement de travaux à l'aiguille de plus en plus prônés dans le cadre du renforcement de l'éducation des filles au travail. ${ }^{11}$

Une enquête réalisée en 1799 auprès des instituteurs de Suisse par le ministre de l'éducation Philipp Albert Stapfer, sous la République helvétique, confirme la présence des élèves-régents des Ecoles de charité dans l'ensemble du canton. Georges Panchaud (1952) en avait retrouvé une quinzaine, qui avaient expressément mentionné la formation aux Ecoles dans leur formulaire de réponse. Grâce aux transcriptions désormais en ligne de cette enquête, ${ }^{12}$ et en comparant la liste de noms d'élèves-régents figurant dans le registre des Ecoles avec celle des instituteurs qui ont répondu à l'enquête, c'est une quarantaine d'anciens élèves qui émerge. Sur 
approximativement 400 postes de maîtres d'écoles élémentaires actives à la fin du siècle ils en occupaient donc grosso modo le $10 \%$. Au nombre de 6 pour la période 1760-1770 (ce qui indique que l'institutionnalisation du séminaire n'a eu aucune influence sur ce point), ils ont passé à 7 à la décennies suivante (1771-1780), puis à 17 (1780-1790), pour arriver à 34 à la fin du siècle (1790-1800), une courbe qui va croissant après la révolution.

Les réponses de ces anciens élèves - dans lesquelles les femmes ont malheureusement, à une exception près, disparu - font apparaître qu'ils écrivaient mieux et avec moins d'erreurs que la moyenne des enseignants vaudois. Et que certains d'entre eux présentent un profil de pédagogue, du moins si l'on neutralise cette catégorie (en lui enlevant nos présupposés) pour l'ouvrir aux représentations des acteurs. C'est le cas de Sébastien Reymond, par exemple, maître dans le village d'Echallens, qui déclare qu' " en été il travaille du menuisier \& tourneur, aime beaucoup la lecture » et qu'il a envoyé aux autorités en 1798 un essai «Sur les Moyens de Perfectionner l'Éducation Nationale ». Aux ouvrages utilisés par la plupart de ses collègues des Ecoles, qui montrent que l'uniformisation des supports d'enseignement entreprise par la Société charitable a eu des répercussions sur l'instruction publique, ${ }^{13}$ Reymond ajoute " une petite grammaire française et la géographie d'Ostervald ».

A plus grande échelle, l'exemple lausannois montre le côté insatisfaisant, parce que réducteur et finaliste, du concept de professionnalisation des enseignants. Echanger les critères empruntés à la sociologie des métiers qui la définissent, contre un concept plus ouvert d'histoire de la profession enseignante, axé sur l'analyse d'une " culture pragmatique " redonne à l'historiographie une fonction de réservoir d'alternatives. Dans cette perspective, fondée sur le rapport à l'altérité plutôt que sur la reconstruction d'une modernité dont nous serions le point d'aboutissement, le maître d'écolecordonnier, cas le plus répandu parmi les maîtres formés aux Ecoles de charité, apparaît moins comme un jalon imparfait sur la voie de la professionnalisation que comme l'incarnation d'un professionnel - muni d'un savoir spécifique, acquis dans une formation préalable assortie d'une expérience pratique - répondant à des attentes sociales. Axées en l'occurrence, comme j'ai essayé de le montrer, sur l'évangélisation, la moralisation et la lutte contre la pauvreté, ces attentes ne faiblissent nullement à la fin du siècle (et peut-être augmentent-elles même tendanciellement en période de crise) d'après les échos qu'en donnent les rapports des Ecoles. ${ }^{14}$

A un autre niveau, le cas lausannois me semble inviter à s'interroger sur le rôle de la religion dans la construction de l'instruction publique, en prenant quelque peu à revers la thèse généralement défendue du passage de l'école des mains de l'Eglise à celle de l'Etat autour de 1800. Une focalisation sur les acteurs qui mobilisent la catégorie « religion " dans leurs actions éducatives ne permettrait-elle pas de dépasser les césures des changements de régimes politiques - dont l'importance reste à mesurer - comme le côté réducteur du binôme Eglise-Etat ? Quelle Eglise ? Quel Etat ? En renonçant à une compréhension essentialiste de ces termes, on ne peut que constater la complexité de ce qu'ils recouvrent. Qu'est-ce que l'Eglise dans les territoires réformés où le "second pouvoir » exercé par ses dignitaires ecclésiastiques a été confisqué par les pouvoirs séculiers ? Si l'Eglise est la communauté d'habitants, comme l'ont 
définie les réformateurs, elle l'est dans la perspective de celles et ceux qui en font partie. L'Eglise catholique - contrairement au clergé - n'existe pas davantage, en dehors d'une perception essentialiste ou des représentations de ceux qui s'en déclarent membres. La religion serait-elle un concept historiographiquement plus saisissable sur une longue durée ? Il y a là un problème à creuser. Laïcs et clercs sont en tous les cas régulièrement associés pour la promouvoir, comme dans le cas des Ecoles de charité ou dans le Réveil religieux des années 1820 et 1830 qui joue un rôle important dans la création des écoles normales. Et combien d'innovations pédagogiques sontelles à mettre au compte de visées religieuses? La liste, en ce qui concerne les Ecoles de charité lausannoises, est longue : outre l'introduction de visites d'écoles trimestrielles, les grilles d'évaluation et leur " pédagogie du progrès " (qui intègre des éléments " lockiens ", tels que les divers degrés d'entendement des enfants, l'émulation opposée aux châtiments), les manuels d'enseignement, dont l'uniformité permit l'enseignement simultané (d'autant que les classes n'excédaient pas quarante élèves), on pourrait encore mentionner la distribution de prix, la tenue de registres des absences et des " fautes capitales " (malheureusement disparus), l'établissement d'une police des écoles (Règles de comportement et de salubrité)... A tous ces égards, les Ecoles de charité, à l'instar d'autres institutions d'évangélisation et de moralisation (songeons aux écoles jésuites), devançaient les écoles publiques. A quels moments l'objectif religieux s'est-il dissous (parfois pour mieux revenir) ou transformé, et dans quoi ?

L'histoire n'est pas la science du passé, comme le rappelait récemment Giovanni Levi dans une conférence à l'université de Lausanne : elle est la science des questions générales avec des réponses particulières, ou locales. Qu'on soit acquis ou non aux changements d'échelles, et à la réduction des espaces d'investigation, il est souhaitable, dans un travail qui ne peut être entrepris que collectivement, de formuler de nouvelles questions, pertinentes pour l'histoire de l'éducation. Un peu à la manière dont l'a fait Ivan Strensky (2003) - auquel j'emprunte le titre de cette conclusion - pour un autre champ relativement jeune de la discipline historique : l'histoire des religions. Mais il convient, en même temps, par un retour à l'archive, de " créer » de nouveaux documents : de nouvelles questions font surgir de nouvelles sources. La recherche sur les egodocuments et l'approche microhistorique ont, au-delà de ce qui les sépare, ceci de commun : leur fort ancrage dans l'archive, voire une véritable « herméneutique de l'archive ». Elles n'en ont bien sûr pas l'exclusivité. Mais, d'une façon ou d'une autre, sans " révolution archivistique " il ne semble pas qu'il puisse y avoir de révolution épistémologique en histoire.

\section{Notes}

1. On pourrait en allonger de beaucoup la liste, avec l'ouvrier du textile Chavatte (Lottin, 1979), le paysan Valentin Jamerey-Duval (Goulemot, 1981), le journalierimprimeur auteur de mémoires dont s'inspira Robert Darnton (1984), Louis Simon, l'étaminier du Maine (Fillon, 1984), l'artisan puritain Nehemiah Wallington (Seaver, 
1985), le scribe Pierre Prion (Le Roy Ladurie \& Ranum, 1985), l'aventurier Jean Conan (Cornette, 1990), le tanneur barcelonais Miquel Parets (Amelang, 1991).

2. Cf. en particulier, parmi de nombreux travaux, les ouvrages collectifs dirigés par Schulze (1996), Greyerz (2001, 2007), Ruggiu (2005, 2013), Ulbrich et al. (2012).

3. Dans l'abondante littérature relative à la microhistoire, je me limite à signaler quelques travaux qui en éclairent les procédés dès les débuts jusqu'à aujourd'hui : Cerutti (2008); Ginzburg (1993); Ginzburg \& Poni (1981); Grendi (2009); Levi (1991); Revel (1996, 2010); Schlumbohn (1998); Schulze (1994).

4. A ce propos voir également Giovanni Levi (1998).

5. Tirés pour le moins à 250 exemplaires à l'intention des sociétaires, du gouvernement bernois et de son représentant à Lausanne, du conseil de la ville de Lausanne et du public intéressé.

6. Prière, catéchisme, lecture et écriture, et, pour les garçons, arithmétique, pour les filles la couture.

7. Cette déclaration est une traduction du point 3 de Methods Used for Erecting Charity Schools (1717).

8. Pour ne donner qu'un exemple : «Pour la lecture : Jeanne Bourillon a paru s'arrêter quelquefois où il ne fallait pas, parler un peu du nez, et lire d'une manière trop monotone. Jeanne Judith Favre a paru lire plus coulamment et avec un peu plus de grâce et de variété de ton ». ([Georges Polier] "Examen et rapport », 1745).

9. «Lavanchy \& Davel sont parvenus assez bien à répondre aux questions qu’on leur a faites mais ils se sont excusés d'interroger eux-mêmes les enfants par jugement n'étant pas faits à cet exercice. » (Polier, «Précis de mon jugement », 1750)

10. Tirée à 3’000 exemplaires, et réimprimée en 1789 (Panchaud 1952, p. 193).

11. Voir notamment le plan pour améliorer l'instruction des filles de FrançoisBarthélémy Ducros, pasteur à Prangins, où il a ouvert une petite école pour les paysans avec le seigneur du lieu : "Dans ces écoles [de filles] on devrait enseigner non seulement à lire, à écrire, et un peu d'orthographe, les règles d'arithmétique, la musique des psaumes, un abrégé de catéchisme, mais encore les ouvrages les plus nécessaires aux filles du peuple, la couture, le tricotage, les formes de raccommodages. [...] J'en connais une placée dans une campagne dont on est parfaitement content à tous égards ; elle est sortie des Ecoles de charité de Lausanne, qui sans doute en pourraient fournir beaucoup d'autres. » (Ducros, 1790).

12. http://www.stapferenquete.ch. Je remercie les collègues responsables de ce projet de m’avoir donné accès aux réponses vaudoises, encore en traitement.

13. "Palettes ou ABC, psaumes en vers, Nouveau Testament et Bible, pour apprendre de mémoire le Catéchisme d'Ostervald retouché, des psaumes, un petit recueil de passages (de l'histoire sainte) ». Notons que sur les 416 catéchismes que mentionne l'" enquête Stapfer » pour le canton de Vaud en 1799, 328 sont des versions, abrégées ou non, du catéchisme d'Ostervald, parmi lesquelles pour le moins 114 correspondent à l'Abrégé retouché pour les Ecoles de charité par Georges Polier, véritable succès de librairie.

14. "Chaque année nous avons la satisfaction de voir quelqu'un de nos élèves 
obtenir quelque régence et porter dans ces utiles mais arides emplois outre une éducation plus soignée, parce qu'elle se prolonge pour eux d'une année au-delà du temps ordinaire, un métier utile aux campagnes et capable de les faire vivre. " (Etat, 1792).

\section{Références}

Amelang, J. (2007). Saving the self from autobiography. In K. von Greyerz (Ed.), Individualisierungsweisen in interdisziplinärer Perspektive (pp. 129-140). München, Germany: R. Oldenbourg.

Amelang, J. (1998). The flight of Icarus: Artisan autobiography in early modern Europe. Stanford, CA: Stanford University Press.

Amelang, J. (1991). A journal of the plague year 1631: The diary of the Barcelona tanner Miquel Parets. New York, NY, and Oxford, England: Oxford University Press.

Becchi, E., \& Julia, D. (1998). Histoire de l'enfance en Occident (Vols. 1-2). Paris, France: Seuil. Bloch, A. (2007). Priester der Volksbildung. Der Professionalisierungsprozess der Zürcher Volksschullehrkeäfte zwischen 1770 und 1914. Zurich, Switzerland: Chronos.

Braggerman, A., \& Dekker, R. (2009). Child of the Enlightenment: Revolutionary Europe reflected in a boyhood diary. Leiden, Netherlands, and Boston, MA: Brill.

Bürgi, A., et al. (1998-2010). Ulrich Bräker, Sämtliche Schriften. München, Germany: H. Beck; Bern, Switzerland: P. Haupt.

Caspard, P. (1996). Education et progrès : ce que disent les écrits personnels. Musée neuchâtelois, 4, 273-289.

Caspard, P. (2006). Singulières ou communes? Les valeurs éducatives révélées par les correspondances entre enfants et parents : Suisse romande et France, 1760-1830. In P. Henry \& J.-P. Jelmini (Eds.), La correspondance familiale en Suisse romande aux XVIIIe et XIXe siècle : affectivité, sociabilité (pp. 31-53). Neuchâtel, Switzerland: Alphil.

Cerutti, S. (2008). Histoire pragmatique, ou de la rencontre entre histoire sociale et histoire culturelle. Tracés. Revue de Sciences humaines, 15, 147-168.

Cornette, J. (1990). Les aventures de Jean Conan. Morlaix, France: Skol Vreizh.

Dahn, N. (2012). L'Abrégé du catéchisme d'Ostervald pour les Ecoles de charité de Lausanne (1750). Entre instruction religieuse et pédagogie des Lumières (Master's thesis). Université de Lausanne, Switzerland.

Darnton, R. (1984). The Great Cat Massacre and other French episodes in French cultural history. New York, NY: Basic Books.

Dekker, R. (2002). Childhood, memory and autobiography in Holland: From the Golden Age to Romanticism. New York, NY: Palgrave Macmillan.

Delumeau, J., \& Roche, D. (Eds.). (1990). Histoire des pères et de la paternité. Mentalités : vécus et représentations. Paris, France: Larousse.

Despoix, P., \& Schöttler, P. (Eds.). (2006). Sigfried Kracauer, penseur de l'bistoire. Paris, France: Editions de la Maison des Sciences de l'Homme, Les Presses de l'Université Laval.

Dosse, F. (1995). L'empire du sens. L’bumanisation des sciences sociales. Paris, France: La découverte. 
Duran Lopez, F. (2009). La autobiografia popular en Espana en los siglos XVIII y XIX : discusion al concepto y aproximacion a un repertorio. In R. Pasta (Ed.), Scritture del io fra pubblico e privato (pp. 225-272). Rome, Italy: Edizione di storia e letteratura.

Ehrenpreis, S. (2010). Histoire de l'éducation et histoire religieuse (France et Saint-Empire, époque moderne). In P. Büttgen \& C. Duhamelle (Eds.), Religion ou confession. Un bilan franco-allemand sur l'époque moderne (XVIe-XVIIIe siècle) (pp. 485-512). Paris, France: Editions de la Maison des Sciences de l'Homme.

Fillon, A. (1984). Louis Simon étaminier (1741-1820) dans son village du Haut-Maine au siècle des Lumières. Le Mans, France: Centre universitaire d'éducation permanente.

Ginzburg, C. (1988). Le fromage et les vers : l'univers d'un meunier du XVTe siècle. Paris, France: Flammarion [Published in Italian: (1976). Il formaggio e i vermi. Il cosmo di un mugnaio del 500. Turin, Italy: Einaudi).

Ginzburg, C. (1993). Microhistory: Two or three things that I know about it. Critical Inquiry, $20,10-35$.

Ginzburg, C., \& Poni, C. (1981, December). La micro-histoire. Le Débat. http://dx.doi. org/10.1086/448699

Goulemot, J.-M. (1981). Valentin Jamerey Duval. Mémoires : enfance et éducation d'un paysan au XVIIIe siècle. Paris, France: Le Sycomore.

Grendi, E. (2009). Micro-analyse et histoire sociale. Ecrire l'histoire, 3, 67-80.

Grendi, E. (1972). Microanalisi e storia sociale. Quaderni Storici, 33, 506-520.

Greyerz, K. von. (2010). Passagen und Stationen. Lebensstufen zwischen Mittelalter und Moderne. Göttingen, Germany: Vandenhoeck \& Ruprecht.

Greyerz, K. von. (Ed.) (2007). Individualisierungsweisen in interdis₹iplinärer Perspektive. München, Germany: R. Oldenbourg.

Greyerz, K. von. (Ed.). (2001). Von der dargestellten Person zum erinnerten Ich : europäische Selbstzengnisse als historische Quellen (1500-1850). Köln, Germany: Böhlau.

Hanafi, N. (2012). Le frisson et le baume. Souffrantes et soignantes au siècle des Lumières (FranceSuisse). (Doctoral thesis, vols. 1-2). Université de Toulouse, France, and Université de Lausanne, Switzerland.

Hardach-Pinke, I. (1981). Kinderalltag. Aspekte von Kontinuität und Wandel der Kindheit in autobiographischen Zeugnissen 1700 bis 1900. Frankfurt, Germany, and New York, NY: Campus.

Hardach-Pinke, I., \& Hardach, G. (Eds.). (1978). Deutsche Kindheiten. Autobiographische Zeugnisse, 1700-1900. Kronberg, Germany: Athäneum.

Jaquet-Francillon, F. (2005). La naissance de la profession d'instituteur. Eléments pour une sociologie historique. Le Télémaque, 28, 49-66. http://dx.doi.org/10.3917/tele.028.0049

Jones, M. G. (1938). The charity-school movement: A study of eighteenth century Puritanism in action. London, England: Cambridge University Press.

Kracauer, S. (1969/2006). History. The last things before the last [L'Histoire des avant-dernières choses]. Paris, France: Stock.

Le Roy Ladurie, E., \& Ranum, O. (1985). Pierre Prion Scribe. Mémoires d'un écrivain de campagne an XVIIIe siècle. Paris, France: Gallimard-Julliard.

Leutert, S., \& Piller, G. (1999). Deutschschweizerische Selbstzeugnisse (1500-1800) als Quellen der Mentalitätsgeschichte. Ein Forschungsbericht. Revue suisse d'bistoire, 49, $197-221$ 
Levi, G. (1989). Le pouvoir au village. Histoire d'un exorciste dans le Piémont du XVIIe siècle. Paris, France: Gallimard. [Published in Italian: (1985). L'eredita immateriale. Carriera di un esorcista nel Piemonte del Seicento. Turin, Italy: Einaudi]

Levi, G. (1989). Les usages de la biographie. Annales. Economies, Sociétés, Civilisations, 44, $1325-1336$.

Levi, G. (1991). On microhistory. In P. Burke (Ed.), New perspectives on historical writing (pp. 93-113). Cambridge, England: Polity Press.

Levi, G. (1998). The origins of modern state and microhistory. In J. Schlumbohn (Ed.), Mikrogeschichte-Makrogeschichte : Komplementär oder inkommensurabel? (p. 55-82). Göttingen, Germany: Wallstein Verlag.

Lottin, A. (1979). Chavatte, owvrier lillois : un contemporain de Lonis XIV. Paris, France: Flammarion.

Macfarlane, A. (1970). The family life of Ralph Josselin, a seventeenth-century clergyman: An essay in historical anthropology. Cambridge, England: Cambridge University Press.

Maissen, T., \& Kempe, M. (2002). Die Collegia der Insulaner, Vertrauliche und Woblgesinnten in Zürich 1679-1709: die ersten deutschsprachigen Aufklärungsgesellschaften zwischen Naturwissenschaften, Bibelkritik, Geschichte und Politik. Zurich, Switzerland: Neue Zürcher Zeitung.

Marx, J. (1971). Autour des 'Pensées philosophiques' : une lettre inédite de Georges Polier de Bottens. Studies on Voltaire and the Eighteenth Century, 84, 99-108.

Moret Petrini, S. (2010). Expérimenter la tenue d'un journal éducatif. Mlle Wittel et le journal de Cécile de Constant. Revue suisse d'Art et d'Archéologie, 67, 296-301.

Panchaud, G. (1952). Les écoles vaudoises à la fin du régime bernois. Lausanne, Switzerland: F. Rouge.

Pasta, R. (Ed.). (2009). Scritture del'io fra pubblico e privato. Rome, Italy: Edizione di storia e letteratura.

Piller, G. (2001). Der jugendliche Männerkörper. Das Jugendtagebuch Johann Rudolf Hubers 1783/84 als Medium der Selbstkontrolle. In K. von Greyerz (Ed.), Von der dargestellten Person, zum erinnerten Ich: europäische Selbstzengnisse als historische Quellen (1500-1850) (pp. 213-230). Köln, Germany: Böhlau.

Pollock, L. A. (1983). Forgotten children: Parent-child relations from 1500 to 1900. Cambridge, England: Cambridge University Press.

Pollock, L. A. (1987). A lasting relationship: Parents and children over three centuries. London, England: Fourth Estate.

Prost, A. (2012). Douze leçons sur l'bistoire. Paris, France: Seuil.

Revel, J. (1989). L'Histoire au ras du sol. In G. Levi, Le pouvoir au village. Histoire d'un exorciste dans le Piémont du XVIIe siècle (pp. I-XXXIII). Paris, France: Gallimard.

Revel, J. (1994). Mircroanalisi e costruzione del sociale. Quaderni Storici, 86, 549-575.

Revel, J. (1996). Micro-analyse et construction du social. In J. Revel (Ed.), Jeux d'échelles. La micro-analyse à l'expérience (pp. 19-36), Paris, France: Gallimard.

Revel, J. (2010). Microstoria. In C. Delacroix, F. Dosse, P. Garcia, \& N. Offenstadt (Eds.), Historiographies, concepts et débats (pp. 529-534). Paris, France: Gallimard.

Roche, D. (1982). Journal de ma vie. Jacques-Louis Ménétra, compagnon vitrier au 18e siècle. Paris, France: Montalba. 
Ruggiu, F.-J. (Ed.). (2013). The uses of first person writing. Les usages des écrits du for privé (Afrique, Amérique, Asie, Occident, Orient). Brussels, Belgium: Peter Lang.

Ruggiu, F.-J., Bardet, J.-P. (Eds.). (2005). Au plus près du secret des ccurs? Nowvelles lectures historiques des écrits du for privé en Europe du XVTe au XVIIIe siècle. Paris, France: PUF.

Schlumbohn, J. (1983). Kinderstuben. Wie Kinder zu Bauern, Bürgern, Aristokraten wurden, 17001850. München, Germany: Deutscher Taschenbuchverlag.

Schlumbohn, J. (Ed.). (1998). Mikrogeschichte-Makrogeschichte: Komplementär oder inkommensurabel? Göttingen, Germany: Wallstein Verlag.

Schulze, W. (Ed.). (1996). Ego-dokumente. Annäherung an den Menschen in der Geschichte. Berlin, Germany: Akademie Verlag. http://dx.doi.org/10.1524/9783050047997

Schulze, W. (Ed.). (1994). Sozialgeschichte, Alltagsgeschichte, Mikrohistorie. Eine Diskussion. Göttingen, Germany: Vandenhoek \& Ruprecht.

Seaver, P. S. (1985). Wallington's world: A Puritan artisan in seventeenth-century London. Stanford, CA: Stanford University Press.

Seibel, V. (1986). Elternhaus, Schule politische Ideen und Erfahrungswelt in der Generation vor und nach 1800. Studien zur Sozialisation in der Umbruchzeit 1770-1850 nach deutschen Autobiographien (Doctoral dissertation). University of Tübingen, Darmstadt, Germany.

Shoemaker, R. B. (1992). Reforming the city: The reformation of manners campaign in London 1690-1738. In L. Davidson et al. (Eds.), Stilling the grumbling hive: The response to social and economic problems in England 1689-1750 (pp. 99-120). Stroud, England: Alan Sutton.

Sonnet, M. (1990). Les leçons paternelles. In J. Delumeau \& D. Roche (Eds.), Histoire des pères et de la paternité (pp. 269-288). Paris, France: Larousse.

Strensky, I. (2003). Why it is better to know some of the questions than all the answers. Method \& Theory in the Study of Religion, 15, 169-186. http://dx.doi. org/10.1163/157006803765218236

Tosato-Rigo, D. (2014). Espace éducatif ou 'chambre à soi' ? Les journaux de Catherine et Angletine Charrière de Sévery. In A. Coudreuse \& C. Seth (Eds.), Le Temps des femmes: Textes mémoriels des Lumières (pp. 69-90). Paris, France: Garnier.

Tosato-Rigo, D. (2012). L'un joue et l'autre pas. Quelques enjeux de l'apprentissage de la lecture en marge de l'enquête Stapfer. Revue historique vandoise, 120, 361-373.

Tosato-Rigo, D. (2009). La chronique de Jodocus Jost, miroir du monde d'un paysan bernois au XVTIe siècle. Lausanne, Switzerland: Société d'histoire de la Suisse romande.

Ulbrich, C. (2007). L'usage historiographique de l'autobiographie. In R. Dion (Ed.), Vies en récit: formes littéraires et médiatiques de la biographie et de l'autobiographie (pp. 139-156).

Montreal, QC: Nota Bene.

Ulbrich, C., Medick, H., \& Schaser, A. (Eds.). (2012). Selbstzengnis und Person : Transkulturelle Perspektiven. Köln and Weimar, Germany, and Vienna, Austria: Böhlau.

Unwin, R. W. (1984). Charity schools and the defence of Anglicanism. York, England: Borthwick Paper 65.

Walter, F. (1997). Une autre histoire sociale. Revue suisse d'bistoire, 47, 59-66. 


\section{Sources manuscrites}

Archives cantonales vaudoises, Chavannes-près-Renens (ACV):

"Liste des garçons élèves dans les Ecoles de charité depuis 1727 à 1755 », 1755

(K XIII 227/11/1)

Listes de garçons et filles des Ecoles de charité avec appréciations (1751-1759)

(K XIII 227/11/2)

[Polier Georges] «Examens et rapport fait des 2 filles prétendant à la place de maîtresse d'école en 1745 "

(KXIII 227/7/2)

[Polier Georges] « Mémoire pour régler les épreuves des prétendants à la place du Sieur Bourillon régent de la 2ème école des garçons et Maître écrivain de toutes les écoles ", 1750

(KXIII 227/7/2)

[Polier Georges] «Précis des Examens à faire des Prétendants à l'emploi de maitre ou mâ̂tresse d'école pour les écoles de charité ", 1750

(KXIII 227/7/2)

[Polier Georges] «Précis de mon jugement sur les épreuves des prétendants à la régence des écoles de charité ", 1750

(KXIII 227/7/2)

Procès-verbaux de la Direction des Ecoles de charité de 1749 à 1760

(KXIII 227/2)

"Registre des Elèves régents des Ecoles de charité de Lausanne dès sa fondation en 1726 à $1804 »$

(K XIII 227/12/1)

Règlement pour le séminaire de régents et maîtresses d'école, 1767

(K XIII 227/6/2)

Bibliothèque de Genève :

Ducros François-Barthélémy, « Observations sur le projet d'améliorer l'éducation de la jeunesse dans la ville de Nyon ", 1790

(Collection Reverdil No 42)

\section{Sources imprimées}

Etat des Ecoles de charité établies à Lausanne. (1727-1802).

Methods used for erecting charity schools, with the rules and orders by which they are governed. (1717).

Polier, G. (1747). Pensées philosophiques chrétiennes mises en parallèle, ou en opposition avec les Pensées philosophiques [de Diderot].

Polier, G. (1747). La liturgie des écoles de charité de Lausanne on le service divin qui s'y fait chaque jour.

Polier, G. (1750). Abrégé du catéchisme d'Ostervald pour les Ecoles de charité de Lausanne.

Relation de l'établissement d'une école de charité dans la ville de Lausanne en 1726.

Talbot, J. (1707). The Christian schoolmaster. 
